\title{
The Humanoid Robot (DARWIN-OP)
}

\author{
S.Vaishnavi ${ }^{1}$, R.Lalitha ${ }^{2}$, B.S Ashwin Prabhakar ${ }^{3}$ \\ Student, Dept. of Computer Technology, Sri Krishna Arts and Science College, Coimbatore, Tamil Nadu, India ${ }^{1,3}$ \\ Assistant Professor, Dept. of Computer Technology, \\ Sri Krishna Arts and Science College, Coimbatore, Tamil Nadu,India ${ }^{2}$
}

\begin{abstract}
The modern era has received a major up blow with the evolution of the robotic domain. The field of robotics is going through major transformation and development. They are already employed for entertainment purposes, assisting the elderly or performing surveillance on small kids. This paper using animations and kinematic tasks, focuses on the implementation and design of the Darwin-OP.
\end{abstract}

Keywords: Sensor, Actuator, Button, Humanoid, Robot

\section{INTRODUCTION}

Humanoids are robots. which perform the function that are assigned like a computer but still look and resemble a human in the looks and structure. This humanoids technology is one of the most welcomed and funded department in the research and development field. This is also called as the anthropomorphic robots. Most of these types of robots are made to work in the human environment as a substitute for them. As these machines are designed in the human form, they'll be more natural to adopt in our daily life than other robots. Not all humanoids will have the entire human anatomy, humanoids will most be constructed like a human arm shaped robot, leg shaped, body above the waist and more. DARwIn-OP (Dynamic Anthropomorphic Robot with Intelligence - Open Platform) is an inexpensive, miniature humanoid robot platform with advance computational power, sophisticated sensors, high payload capacity, and dynamic motion ability to enable many exciting research, education, and outreach activities. To solve this iterative problem, a humanoid must be constructed considering expandable-modifiable system structure, high performance, simple maintenance, familiar development environment, and affordable prices. Therefore, in this paper, the design for humanoid platform DARwIn-OP which has a network based standardized structure and a standard PC architecture to meet above requirements.

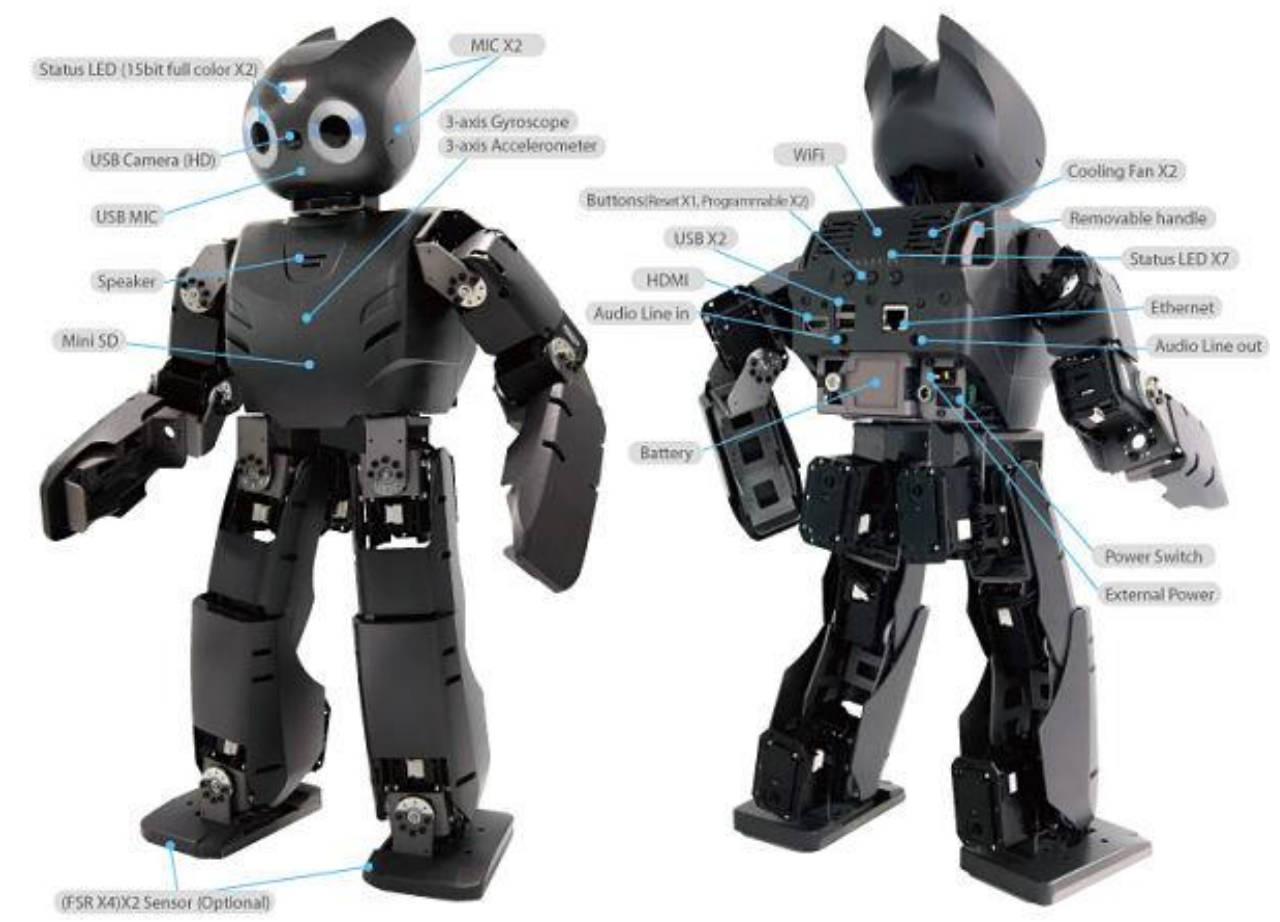

The typical DARWIN-OP is shown in fig.1 


\section{IJARCCE}

Vol. 7, Issue 11, November 2018

\section{SYSTEM OVERVIEW}

Fig. 2 shows us the modular structure which is completely network based and has a standard PC architecture. All devices like sensors, LED's, buttons, actuators and external I/Os that are connected to the sub controller by serial bus network which is completely supported by DYNAMIXEL protocol. Each device has its own memory mapped operation with its own designated ID. For the main controller, we adopt the Intel's ATOM Z530 CPU which is usually used for net books. The main controller and the sub controller communicates through USB(Universal Serial Bus). To access the device the sub controller works as a gateway. Therefore all the devices are enclosed as an USB device, that means that the development environment is just like a standard PC.

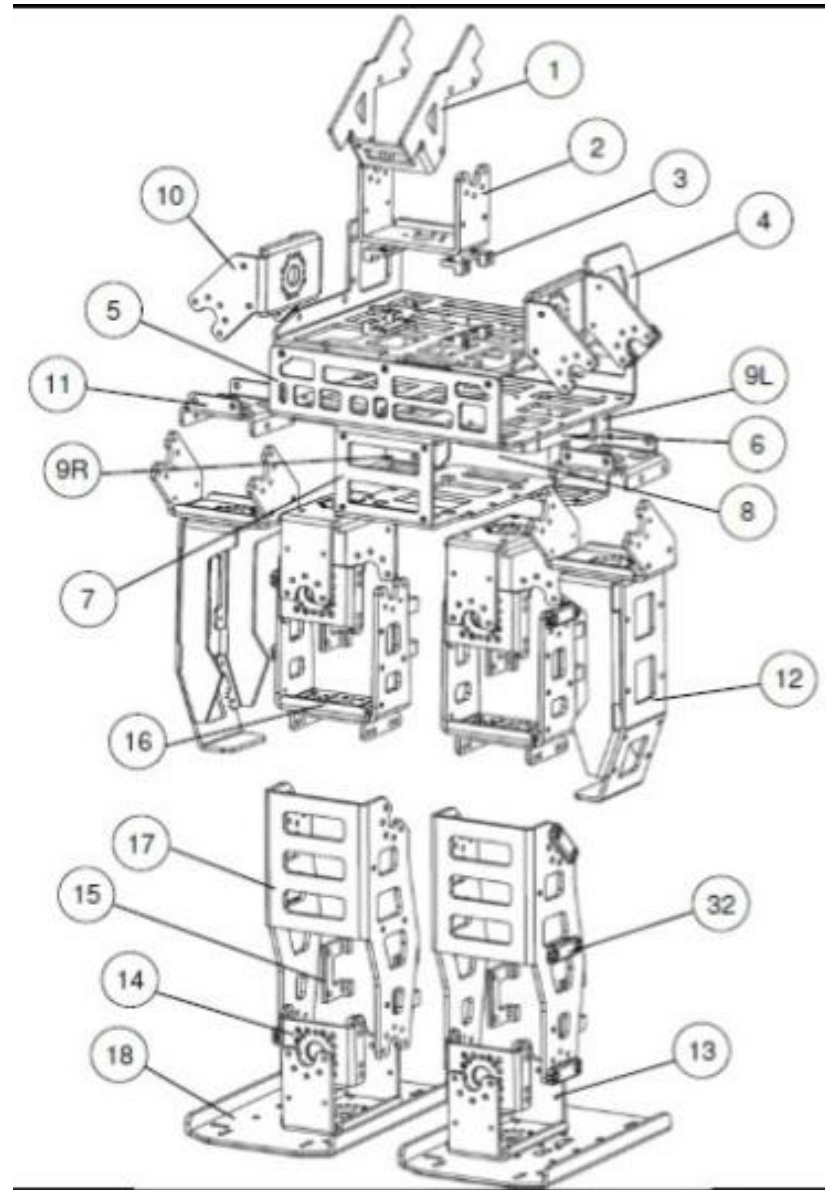

\begin{tabular}{|c|c}
\hline Part Number & Description \\
\hline 1 & Head Bracket \\
\hline 2 & Neck Bracket \\
\hline 3 & Chest Actuator Connectors \\
\hline 4 & Upper Chest Bracket \\
\hline 5 & Lower Chest Bracket \\
\hline 6 & Mounting Bracket \\
\hline 7 & Battery Bracket \\
\hline 8 & Battery Cover Bracket \\
\hline 9 L & Back Left Pelvic Bracket \\
\hline $9 R$ & Back Right Pelvic Bracket \\
\hline 10 & Angled Actuator Bracket \\
\hline 11 & Arm Actuator Mount \\
\hline 12 & Hand \\
\hline 13 & U-Actuator Bracket \\
\hline 14 & Leg Actuator Mount \\
\hline 15 & Actuator Connector \\
\hline 16 & Thigh Bracket \\
\hline 17 & Knee Bracket \\
\hline 18 & Foot \\
\hline 32 & Cable Holder \\
\hline
\end{tabular}

Fig. 2DARwIn-OP System

\section{HARDWARE STRUCTURES}

Like it is represented on figure 2.1. The DARwIn-OP has two controllers. The most important one is SBC - fit PC 2 on which Ubuntu 9.10 is running. It is equipped with $1 \mathrm{~GB}$ of RAM , a CPU of $106 \mathrm{GHz}$ and a flash disk of 4GB.It also has a HDMI video out, 2 USB ports, an Ethernet port, Wi-Fi and 2 audio ports (IN and OUT). The sub-controller is a CM-730 that was manufactured by Robotics. Most of the sensors and actuators are connected to the sub-controller. An actuator is a part of a robot that is used for moving and controlling a system or robot.The controller has to send or ask the values to the sub-controller and it is in charge of reading the state of the requested sensors or in state of applying the requested values to the actuator.

Only a HD Logitech C905 Camera of 2M pixels is connected directly to the main controller.

The sensors given below are connected to the sub-controller:

- A 3 axes gyroscope.

- A 3 axes accelerometer.

- 3 push buttons.

- 2 microphones.

- $\quad$ Optionally 8 pressure meters (4 under each foot). 


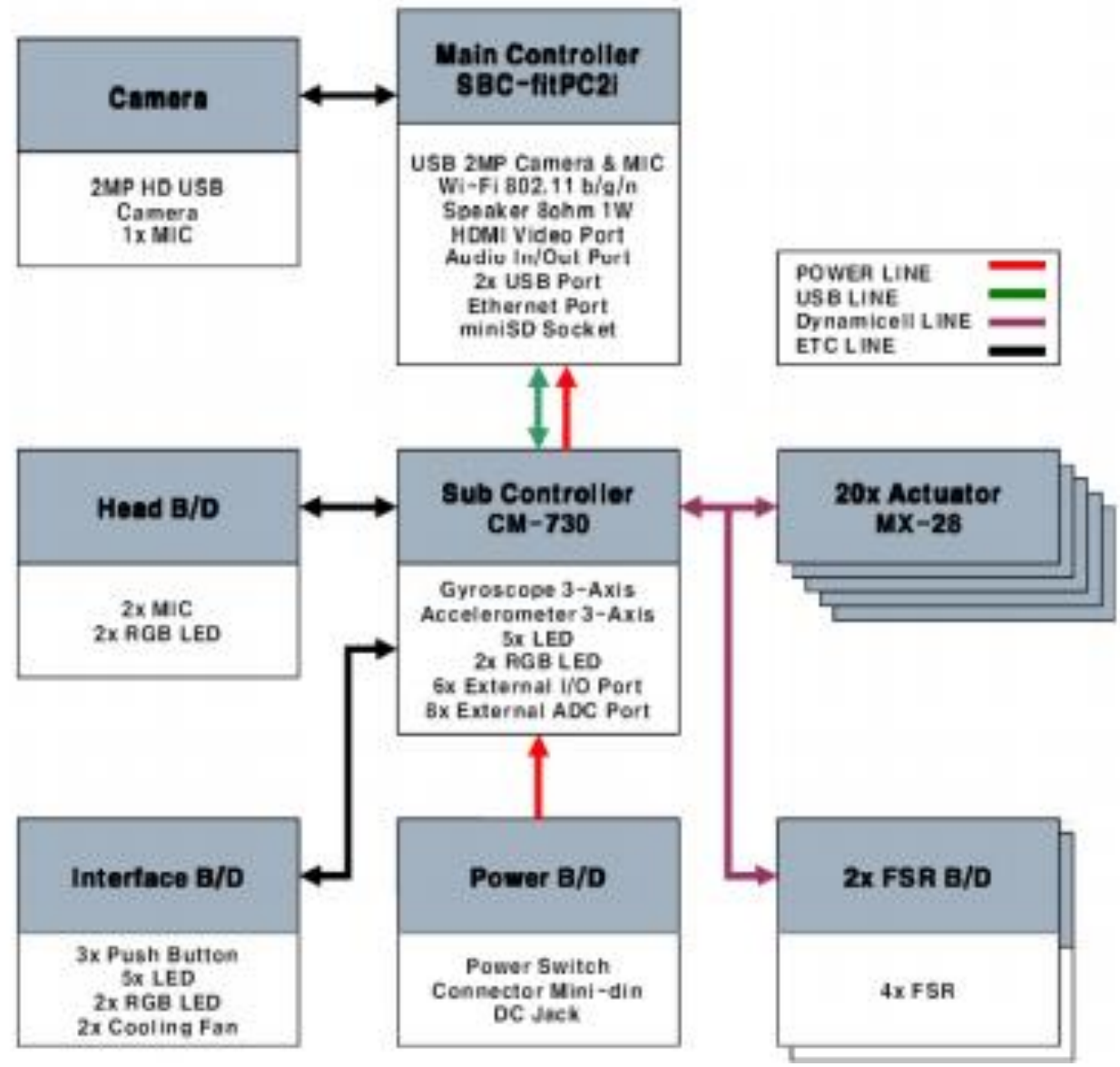

Fig. 2.1 Hardware Structure of the DARwIn-OP

\section{SYSTEM INTEGRATION}

\section{Control architecture}

KHR-1(Kondo Humanoid Robot) was developed in 2002, the central control system was introduced. So a main computer with DOS (Disk Operating system) skillful all joints by using an interface card. All agents run concurrently and handle minimum necessary input data. Once the condition for agent supplication is satisfied, the agents become active and work on achieving their tasks. Selecting standards are fast CPU speed, low power consumption, compact size and expansion interface.

\section{CAN (Controller Area Network) protocol}

In order that the main computer can give orders to sub controllers or receive the many types of data, there must be communication bus line between the main computer and the sub controller. Also the communication speed should be fast to handle 19 sub controllers. Therefore we implemented the CAN protocol which is high speed serial communication up to $1 \mathrm{Mbit} / \mathrm{s}$. The presentation of CAN protocol has already been proved in automobile industry. In CAN protocol, just two lines are wanted for transmission of data, so it is very simple to expand other sub controllers. The most significant feature of CAN is a Multi-Master/Multi Slave feature. This means that all controllers linked with the CAN bus line can be Master, so they can transmit any data to CAN bus line. And then, all controllers can receive data in CAN bus at the similar time. So if the main controller sends the data to the CAN bus line, every controller can obtain the data.

\section{Sub controller}

There are two types of sub controllers. The one is Joint Motor Controller(JMC) and the other is sensory device. All sub controllers were intended by ourselves and their MPU(Micro Processor Unit) are the same. This MPU has a CAN module and connects with a main computer. Each controller also has numerous the A/D(Analog to Digital) converter, so we can easily add sensors. 


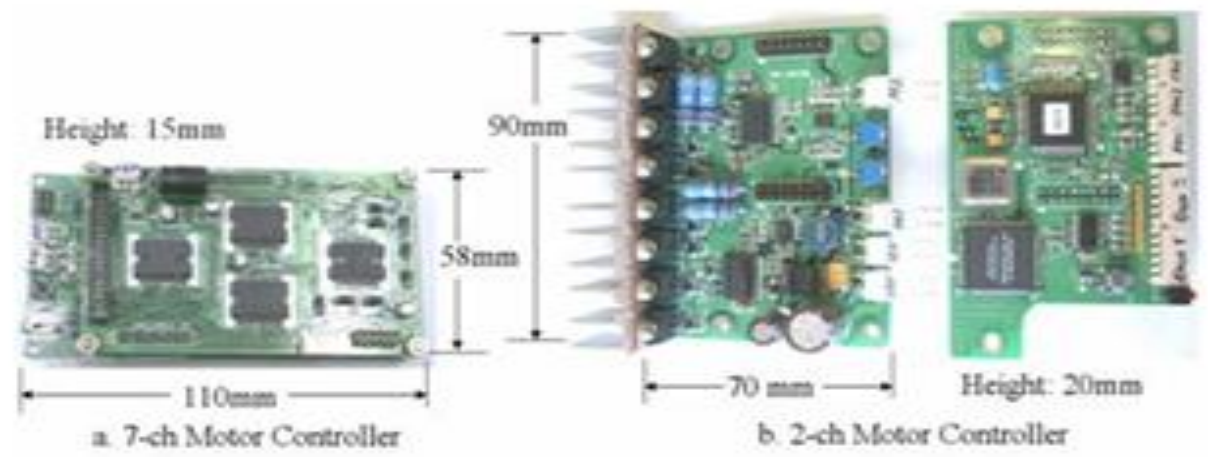

\section{Joint motor controllers}

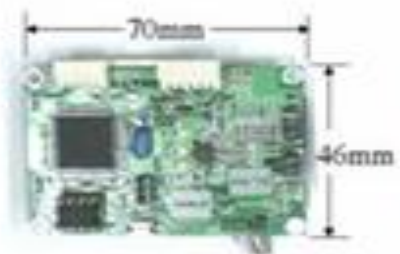

a. Signal Procesung Modula

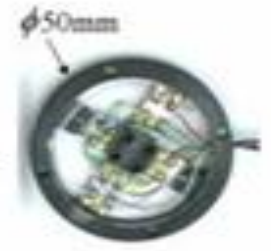

b. FrT Senser in Wrist

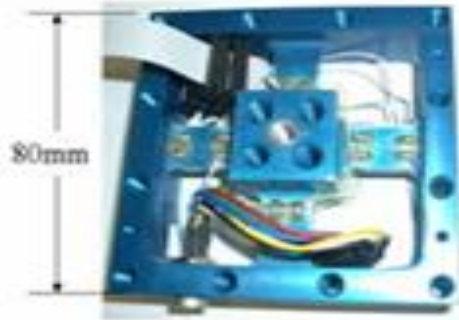

c. F/T Semor an Arable

\section{Axis Force/Torque sensors}

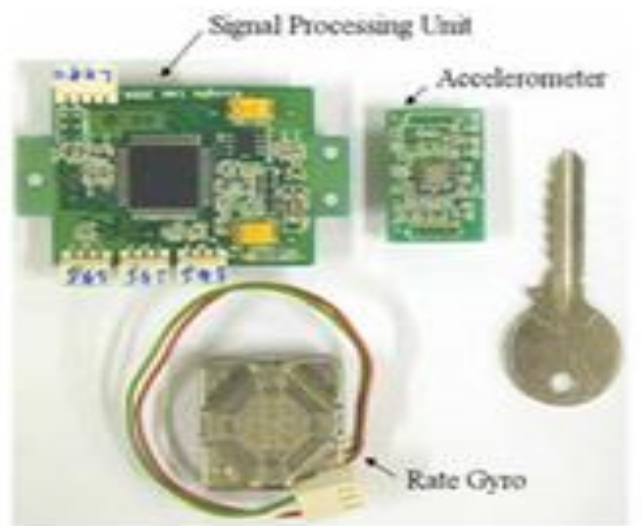

\section{Real-time operating system of the main computer}

It is a General Purpose Operating System (GPOS). So user-mode application program can not have a admission to access the hardware directly. And because Windows thread scheduler is not deterministic, its interject latency may be over 5 msec. Therefore we used the RTX(Real Time Extension) which is a HAL(Hardware Abstraction Layer) extension commercial program in order to deliver real-time capability. By using RTX, we can admission hardware directly. Also we can make an interject which has the highest priority and its maximum latency is just 12 usec. In this way, we provided the real-time capability to the main computer easily, and were able to grow the main control program fast in Windows environment by using Visual C++ language.

\section{SOFTWARE STRUCTURES}

The software of DARwIn-OP has built with a hierarchical framework considering modularity and independency. These framework consists of device communication module, motion module, walking module, sensing module, behavior module, vision module, and diagnostics module. The framework is developed with $\mathrm{C}++$ programming language which is OS independent. The OS independent aspect is essential so that it can be ported to any of the existing or future computer systems it also includes newly-developed Robot Operating System (ROS). There is no need to develop a separate 
framework set, the user simply can write a behavioral code for DARwIn-OP. In such of these cases software simulator is the most practical method for writing and testing the program. The users are encouraged to share the developed coding to the other users as the DARwIn is a open-source. There are currently many independent-software that do not implement open- DARwIn-SDK. The low-level programming takes care of the robot's sub-routines the refresh time of camera or to read the actuator's position. The high-level programming can take care of the most important aspect of the robots.

\section{APPLICATIONS}

The above proposed project helps and will have a revolutionary impact in the field of developing the humanoid robots the domain feasibility of the project is also high.

$>\quad$ There is a one-to-one mapping between the human and the humanoid body.

$>$ Humanoid robots take advantage of human environments and equipment.

$>$ Humanoid robots are easier to train.

$>$ Teamwork is easier between humans and humanoids.

$>\quad$ The locals could potentially interact with humanoid robots.

\section{WALKING ALGORITHM}

In this section, we introduce the basic active walking algorithm. At the first stage, user has to set numerous walking parameters. They are step length, step period, double support phase ratio, lateral swing largeness of body center and amplitude of foot lift and so on. At the second stage, user selects the walking types such as forward or backward walking, right/left side walking and clockwise/ counter clockwise turning. At the third stage, proper walking design is generated according to the walking parameters and walking types. And walking pattern is adapted by trunk roll/pitch controller and landing position controller. Trunk roll/pitch controller is used to prevent the proclivity of the trunk from the change of the ground proclivity by using rate gyro and accelerometer. And landing position controller stops the unstable landing by modification of position schedule, when the actual landing occurs before or after the set time.

At the fourth stage, all joints angles are resulting by inverse kinematics and then, also modified by checking controller and landing orientation controller. These controllers are switched each other by landing detection algorithm. We removed the sustained vibration in single support phase by damping control at ankle joint which is based on torque feedback. And for soft landing, we applied the mooring orientation controller at ankle joint which is based on integral of torque.

Finally, the main computer sends the orientation position data to all joint motor controllers and then all joint motor controllers control the DC motors by using PD control. In this way, we did the walking experiments. The stride was $0 \sim$ $60 \mathrm{~cm}$ and KHR-2(Kaist Humanoid Robot) walked straightly on the treadmill with $0 \sim 1.0 \mathrm{~km} / \mathrm{h}$. And it was able to walk to right or left side with $0 \sim 80 \mathrm{~cm}$ of side step length and turn to counter clockwise or clockwise with $0 \sim 15 \mathrm{deg}$ a step.

\section{CONCLUSION}

Thus it is an innovative idea to help the people who can't do their work themselves and need someone help. I have discussed Walking Module in this journal. The remaining modules will be discussed in future journal.

Table I. Overall specifications

\begin{tabular}{ccc}
\hline Category & Description & Data \\
\hline \multirow{2}{*}{ Dimension } & Height & $0.455 \mathrm{~m}$ \\
& Weight & $2.8 \mathrm{Kg}$ \\
\hline \multirow{3}{*}{ DOF } & Head & 2 DOF \\
& Arm & $2 \times 3$ DOF \\
& Leg & $2 \times 6$ DOF \\
\hline \multirow{4}{*}{ Main } & CPU & Intel Atom Z530@1.6GHz \\
Controller & RAM & $1 \mathrm{~GB}$ DDR2 \\
& Disk & 4 GB Flash Disk \\
& Network & Ethernet/WiFi \\
& USB Port & $2 \times$ USB2.0 \\
\hline
\end{tabular}




\section{IJARCCE}

International Journal of Advanced Research in Computer and Communication Engineering

Vol. 7, Issue 11, November 2018

\begin{tabular}{|c|c|c|}
\hline \multirow{4}{*}{$\begin{array}{c}\text { Sub } \\
\text { Controller }\end{array}$} & $\mathrm{CPU}$ & ARM 32-bit Cortex-M3 \\
\hline & Frequency & $72 \mathrm{MHz}$ \\
\hline & Flash Memory & $512 \mathrm{~KB}$ \\
\hline & SRAM & $64 \mathrm{~KB}$ \\
\hline \multirow{5}{*}{$\begin{array}{l}\text { Actuator } \\
\text { MX-28 }\end{array}$} & Holding Torque & $24 \mathrm{kgfem} @ 12 \mathrm{~V}$ \\
\hline & Speed & 45RPM@ No Load \\
\hline & Position Sensor & Magnetic Potentiometer \\
\hline & Resolution & $0.072^{\circ}$ \\
\hline & Command Interface & Serial 3MBPS \\
\hline \multirow{4}{*}{ Sensor } & Gyroscope & 3-Axis \\
\hline & Accelerometer & 3-Axis \\
\hline & Pressure-meter & $2 \times 4$ FSR in Foot \\
\hline & Camera & 2MP HD USB \\
\hline \multirow{4}{*}{ Software } & $\mathrm{O} / \mathrm{S}$ & Linux Ubuntu \\
\hline & Framework & open-DARwIn SDK \\
\hline & Language & $\mathrm{C}++/$ Java \\
\hline & Compiler & GCC \\
\hline
\end{tabular}
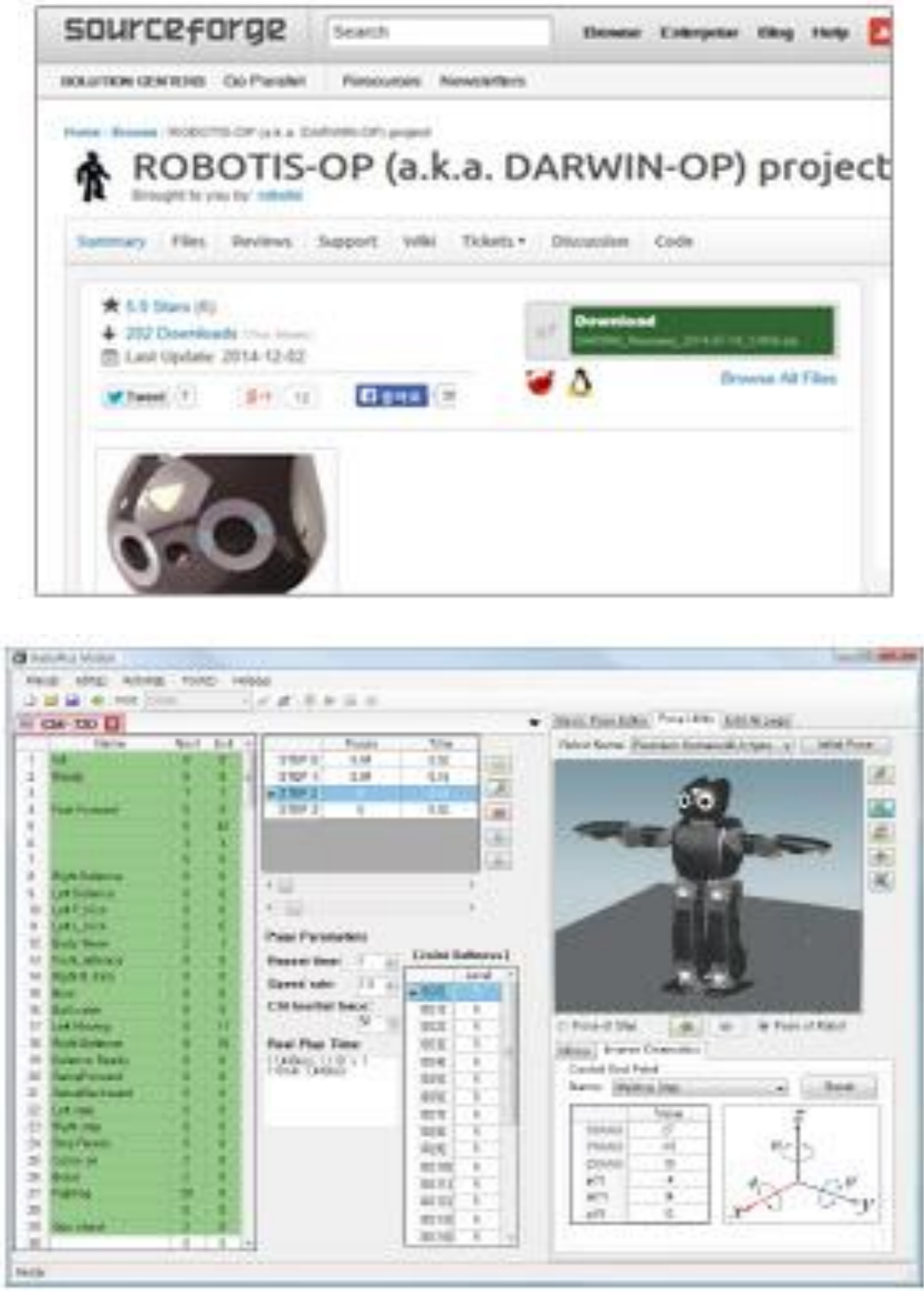


\title{
IJARCCE
}

\author{
Vol. 7, Issue 11, November 2018
}

\section{REFERENCES}

[1]. K. Hirai, M. Hirose, Y. Haikawa, and T. Takenaka, "The Development of Honda Humanoid Robot",International Conference on Robotics and Automation, pp. 1321-1326, 1998

[2]. Inyong Ha, Yusuke Tamura, Hajime Asama, Jeakweon Han and Dennis W Hong “ Department of Precision Engineering” SICE Annual Conference 2011 September 13-18, 2011.

[3]. http//:robotics.gatech.edu/hg/item/215691

[4]. http://www.seas.upenn.edu/media/fteaure-darwin.php

[5]. S Behnke, Humanoid Robot, Humanoid Robot - From Fiction to Reality, 2008, 4(8), 5.

[6]. H Televox, The Robots of Westinghouse, History of Computers, Webhttp://history-computer.com/Dreamers/Elektro.html

[7]. A Takanishi, Humanoid Robotics Research and Its Applications, Hadaly-2 and WABIAN, Humanoid Robots in Waseda University, 2002, 12(1), 25-38.

[8]. C Run Bin, Inverse Kinematics of a New Quadruped Robot Control Method, International Journal of Advanced Robotic Systems, 2013 , $10(1), 2-5$.

[9]. JR Movellan, Intelligent Robotics and Communications, Origins, Principles, and First Steps, The RUBI/QRIO Project, 2005, $223-228$.

[10]. 10]HS Ahn and JY Choi, Can We Teach What Emotion a Robot Should Express?, 2012, 1407-1412.

[11]. J Pratt, Virtual Model Control, An Intuitive Approach for Bipedal Locomotion, 2001, 20(2), 129-130.

[12]. M Vukobratovic and B Borovac, Humanoid Robotics, Biological Principles of Control Selection for a Humanoid Robot's Dynamic Balance Preservation, 2008, 5(4), 642-646.

[13]. T Mcgeer, Dynamics and Control of Bipedal Locomotion, 1992, 290-295.

[14]. J Vaganay, Mobile Robot Attitude Estimation, Mobile Robot Attitude Estimation by Fusion of Inertial Data, 1993, 1(2), $277-282$.

[15]. DY Lee, 3D Vision Based Obstacle Avoidance Method, 2012, 473-475.

[16]. H Bay, Speeded up Robust Feature (SURF), Computer Vision and Image Understanding, 2008, 110(3), $346-359$.

[17]. U Farooq, Fuzzy Logic and Obstacle Avoidance, Fuzzy Logic Based Real Time Obstacle Avoidance Controller for a Simplified Model of Hexapod Walking Robot, 2014, 6(2), 127-130.

[18]. K Ichiro, The Robot Musician, Robotics, 1987, 3(2), 143-155.

[19]. F Ferland, Natural Interaction Design of a Humanoid Robot, Journal of Human-Robot Interaction, 2012, 1(2), 119-130. 